\title{
Desempenho de Trichogramma galloi (Hymenoptera: Trichogrammatidae) sobre ovos de Diatraea spp. (Lepidoptera: Crambidae)
}

\author{
Ellen Carine Neves Valente(1), Sônia Maria Forti Broglio(1), \\ Eliana Maria dos Passos ${ }^{(2)}$ e André Suêldo Tavares de Lima ${ }^{(3)}$
}

\begin{abstract}
(1)Universidade Federal de Alagoas, Centro de Ciências Agrárias, BR 104 Norte, Km 85, CEP 57100-000 Rio Largo, AL, Brasil. E-mail: ellencvalente@yahoo.com.br, soniamfbroglio@gmail.com ${ }^{(2)}$ Embrapa Tabuleiros Costeiros, Avenida Beira Mar, no 3.250, Jardins, CEP 49025-040 Aracaju, SE, Brasil. E-mail: bisologa@hotmail.com ${ }^{(3)}$ Instituto Federal de Educação, Ciência e Tecnologia de Alagoas, AL 101 norte, s/no, Centro, CEP 57955-000 Maragogi, AL, Brasil. E-mail: andresueldo@ig.com
\end{abstract}

Resumo - O objetivo deste trabalho foi avaliar o desempenho de Trichogramma galloi (Hymenoptera: Trichogrammatidae) sobre ovos de Diatraea flavipennella e D. saccharalis (Lepidoptera: Crambidae), assim como as características biológicas do parasitoide em ambos os hospedeiros, e elaborar a tabela de vida de fertilidade. Para isso, foram realizados testes com e sem chance de escolha e bioensaios para avaliar as características biológicas do parasitoide em ambos os hospedeiros. A tabela de vida de fertilidade foi elaborada por meio do parasitismo diário das fêmeas de $T$. galloi sobre os hospedeiros. Os resultados da percentagem de parasitismo em testes com chance de escolha mostraram uma melhor aceitação por parte de T. galloi quanto a ovos de $D$. flavipennella. Com relação aos parâmetros biológicos do parasitoide em ambas as espécies, não houve diferença significativa. Resultados da tabela de vida de fertilidade evidenciam que $D$. flavipennella e D. saccharalis permitem aumento populacional para T. galloi. O parasitoide T. galloi apresenta desempenho satisfatório em ambos os hospedeiros, sendo um potencial agente de controle para essas espécies.

Termos para indexação: broca-da-cana-de-açúcar, chance de escolha, controle biológico, parasitoide, tabela de vida.

\section{Performance of Trichogramma galloi (Hymenoptera: Trichogrammatidae) on eggs of Diatraea spp. (Lepidoptera: Crambidae)}

\begin{abstract}
The objective of this work was to evaluate the performance of Trichogramma galloi (Hymenoptera: Trichogrammatidae) on eggs of Diatraea flavipennella and D. saccharalis (Lepidoptera: Crambidae), as well as the biological characteristics of the parasitoid in both hosts, and to elaborate the fertility life table. For this, tests with and without choice and bioassays to evaluate the biological characteristics of the parasitoid in both hosts were performed. The fertility life table was developed through daily parasitism of $T$. galloi females on the hosts. The percentage results of parasitism in free-choice tests showed a better acceptance by $T$. galloi concerning eggs of $D$. flavipennella. Regarding the biological parameters of the parasitoid in both species, there was no significant difference. Fertility life table results show that D. flavipennella and D. saccharalis allow population increase for $T$. galloi. The parasitoid $T$. galloi has satisfactory performance in both hosts, and is a potential control agent for these species.
\end{abstract}

Index terms: sugarcane borer, free choice, biological control, parasitoid, fertility life table.

\section{Introdução}

As brocas Diatraea saccharalis (Fabricius, 1794) e Diatraea flavipennella (Box, 1931) (Lepidoptera: Crambidae) são importantes pragas da cultura da cana-de-açúcar no Brasil. A primeira espécie apresenta ampla distribuição geográfica no País, enquanto a segunda se restringe basicamente aos canaviais nordestinos (Cheavegatti-Gianotto et al., 2011).
O controle dessas brocas vem sendo realizado por meio do emprego de cultivares resistentes, bem como liberações do endoparasitoide larval Cotesia flavipes (Cameron, 1891) (Hymenoptera: Braconidae) (Dinardo-Miranda, 2008; Dinardo-Miranda et al., 2012; Lima et al., 2013). No entanto, em áreas onde a predação de ovos é baixa, tem-se recomendado o uso do parasitoide de ovos Trichogramma galloi Zucchi, 1988 (Hymenoptera: Trichogrammatidae) (Botelho 
et al., 1999). No Brasil, esse parasitoide é produzido em escala industrial e liberado em cerca de $500 \mathrm{mil}$ hectares com cana-de-açúcar (Vasconcelos, 2012).

O emprego de T. galloi apresenta grande importância para as lavouras da cana-de-açúcar no Brasil, uma vez que esses parasitoides parasitam os ovos das brocas, $\mathrm{o}$ que impede sua progressão na cultura, bem como por serem facilmente multiplicados em laboratório (Oliveira et al., 2014). Além disso, na região Nordeste, onde há predominância de D. flavipennella, estudos acerca do desempenho de T. galloi sobre essa espécie, e que os relacionem com sua espécie similar, $D$. saccharalis, são escassos (Freitas et al., 2007; Dinardo-Miranda et al., 2010). Uma das causas pelas quais estudos com D. flavipennella são reduzidos é a dificuldade de manutenção da criação em laboratório, uma vez que essa espécie apresenta um ciclo longo e desuniforme.

Parasitoides do gênero Trichogramma apresentam variadas características que podem influenciar no sucesso das liberações, o qual se baseia, entre outros fatores, no conhecimento das características bioecológicas do parasitoide e da sua interação com o hospedeiro alvo (Siqueira et al., 2012).

Informações acerca da preferência de parasitoides, por exemplo, são relevantes em virtude da ocorrência de mais de uma espécie hospedeira, situação que pode interferir no desempenho do parasitoide em suprimir o número de insetos de uma praga-alvo, em virtude da maior oferta de recurso alimentar para prole (Siqueira et al., 2012). Para avaliar a preferência de parasitoides, são utilizados testes sem chance de escolha, em que cada espécie é oferecida separadamente, e com chance de escolha, em que ambas as espécies são oferecidas simultaneamente (Hassan, 1997).

O objetivo deste trabalho foi avaliar o desempenho de Trichogramma galloi (Hymenoptera: Trichogrammatidae) sobre ovos de Diatraea flavipennella e D. saccharalis (Lepidoptera: Crambidae), assim como as características biológicas do parasitoide em ambos os hospedeiros, e elaborar a tabela de vida de fertilidade.

\section{Material e Métodos}

Os experimentos foram realizados no Laboratório de Entomologia Agrícola, do Centro de Ciências Agrárias, da Universidade Federal de Alagoas (Ceca/ Ufal), em Rio Largo, AL (12 $40^{\prime}$ S, 3906' W, 127 m de altitude) de abril a setembro de 2014. O local do experimento foi climatizado a $25 \pm 2^{\circ} \mathrm{C}$, UR de $70 \pm 10 \%$ e fotofase de 12 horas.

O parasitoide Trichogramma galloi foi adquirido da criação estoque do Laboratório de Biologia de Insetos da Escola Superior de Agricultura Luiz de Queiroz, Piracicaba, SP. Esses parasitoides foram cedidos também pela empresa BUG Agentes de Controle Biológico (Piracicaba, SP), na qual a criação é realizada em ovos de Anagasta kuehniella (Zeller, 1879) (Lepidoptera: Pyralidae). A criação foi mantida em tubos de fundo chato $(8,5 \times 2,4 \mathrm{~cm})$, vedados com filme PVC com um orifício para aeração. Para alimentação dos adultos do parasitoide, depositou-se uma gotícula de mel no interior do recipiente. Para a manutenção do parasitoide, foram utilizados ovos de $D$. saccharalis, por quatro gerações. Por ocasião dos testes com e sem chance de escolha, utilizaram-se parasitoides oriundos de ovos de A. kuehniella.

As espécies $D$. flavipennella e $D$. saccharalis foram obtidas, no estágio de pupa, do Laboratório de Controle Biológico da Usina Santo Antônio em São Luiz do Quitunde, AL (9 $28^{\prime} \mathrm{S}, 35^{\circ} 56^{\prime} \mathrm{W}, 127 \mathrm{~m}$ de altitude). As pupas foram acondicionadas em recipiente plástico (26x17x8 cm), com o fundo forrado com papel filtro sobre uma camada de algodão umedecido, até a emergência dos adultos.

Os adultos foram mantidos em gaiolas de PVC, medindo $20 \times 22 \mathrm{~cm}$ (diâmetro $\mathrm{x}$ altura), revestidas internamente com papel sulfite, que serviu de substrato para as posturas. No interior dessas gaiolas foi oferecida solução de mel (5\%) e água como alimento aos adultos. Elas foram mantidas a $23 \pm 2^{\circ} \mathrm{C}$, UR de $70 \pm 10 \%$ e fotófase de 12 horas.

Os ovos obtidos foram desinfestados com soluções de formol $(3 \%)$ e sulfato de cobre $(1 \%)$, sendo em seguida armazenados em câmara úmida até a eclosão das lagartas $\left(25 \pm 2^{\circ} \mathrm{C}\right.$, UR de $70 \pm 10 \%$ e fotófase de 12 horas). As posturas utilizadas nos bioensaios não passaram por esse procedimento.

As lagartas recém-eclodidas foram distribuídas em tubos de vidro com dieta artificial. Esse procedimento foi adotado apenas para a criação de $D$. flavipennella, uma vez que a viabilidade de ovos dessa espécie é reduzida quando estes são transferidos diretamente para a dieta. $\mathrm{Na}$ criação de $D$. saccharalis isso não ocorreu, tendo as posturas sido diretamente transferidas para a dieta. Após 15 dias, as lagartas foram colocadas 
em placas de Petri plásticas $(6,0 \mathrm{~cm}$ de diâmetro $\mathrm{x}$ $1,3 \mathrm{~cm}$ de altura) com dieta, as quais foram revisadas, periodicamente, para retirada de pupas. A dieta artificial utilizada para alimentação das lagartas de Diatraea sp. foi constituída basicamente de farelo de soja, germe de trigo, açúcar, sais de Wesson, ácido ascórbico, solução vitamínica, água e anticontaminantes (Macedo, 2002).

Nos experimentos com e sem chance de escolha, foram utilizadas fêmeas de $T$. galloi com 12-24 horas de idade, provenientes de ovos de A. kuehniella, evitando-se, assim, o condicionamento pré-imaginal (Siqueira et al., 2012). Para os testes foram oferecidas posturas de 12 a 24 horas de idade de D. flavipennella e D. saccharalis às fêmeas de T. galloi. As posturas continham de 15 a 20 ovos, os quais foram acondicionados em tubos de vidro de fundo chato $(8,5 \times 2,4 \mathrm{~cm})$ com uma fềmea do parasitoide e uma gotícula de mel para alimentação. Os tubos foram fechados com filme de PVC e mantidos em sala climatizada a $25 \pm 2^{\circ} \mathrm{C}, 70 \pm 10 \%$ de UR e 12 horas de fotófase. Após 24 horas de parasitismo, as fêmeas foram retiradas dos tubos, e os ovos avaliados a partir do sétimo dia.

Nos testes com chance de escolha, posturas dos dois hospedeiros foram expostas ao parasitismo simultaneamente (D. flavipennella, na presença de $D$. saccharalis, e D. saccharalis, na presença de D. flavipennella). Nos testes sem chance de escolha, os ovos foram oferecidos separadamente às fêmeas de T. galloi (D. flavipennella sem chance de escolha $\mathrm{x}$ $D$. saccharalis sem chance de escolha).

$\mathrm{O}$ experimento foi realizado em delineamento inteiramente casualizado, composto por dois tratamentos (D. Alavipennella e D. saccharalis) com 15 repetições cada um e uma fêmea por repetição. Diferenças dos parâmetros biológicos entre os hospedeiros foram determinadas utilizando o teste $\mathrm{F}$, a $5 \%$ de probabilidade.

Os seguintes parâmetros foram avaliados: número de ovos parasitados, percentagem de emergência, razão sexual e número total de descendentes. A razão sexual foi calculada com a divisão do número de fêmeas pelo total de indivíduos (machos + fêmeas) (Silveira-Neto et al., 1976), sendo o dimorfismo sexual determinado pelas antenas, as quais nas fêmeas são clavadas e nos machos são plumosas.

Para avaliação das características biológicas de T. galloi sobre D. flavipennella e D. saccharalis, fêmeas de T. galloi provenientes de ovos de A. kuehniella foram transferidas, individualmente, para tubos de vidro de fundo chato $(8,5 \times 2,4 \mathrm{~cm})$ com uma gotícula de mel e vedadas com filme PVC. Posturas de D. saccharalis e D. flavipennella foram oferecidas, individualmente, às fêmeas com 24, 48 e 72 horas de idade, por um período de 24 horas, após o qual as posturas foram retiradas e avaliadas conforme o bioensaio de preferência. Fêmeas provenientes dos ovos de ambas as espécies foram alimentadas com uma gotícula de mel para avaliação da mortalidade. $O$ experimento foi realizado em delineamento inteiramente casualizado, em esquema fatorial $2 \times 3$ (sendo dois hospedeiros $-D$. flavipennella e $D$. saccharalis -; e três idades de fêmeas - 24,48 e 72 horas), com 15 repetições.

Com base nos dados obtidos pela mortalidade das fêmeas, determinou-se a sobrevivência média, tendo os dados sido submetidos ao teste de Log-Rank, por pares de isolados, pelo método Kaplan-Meyer, com auxílio do Proc Lifetest do programa SAS (SAS Institute, Cary, NC, EUA)).

Os demais dados obtidos nos testes foram submetidos à análise de variância (Anova), e as médias comparadas pelo teste de Tukey a $5 \%$ de probabilidade. Para todas as análises, utilizou-se o programa estatístico SAS (SAS Institute, Cary, NC, EUA).

Para elaboração da tabela de vida e fertilidade, 20 fêmeas de $T$. galloi com 24 horas de idade, provenientes de ovos do hospedeiro alternativo $A$. kuehniella, foram individualizadas em tubos de vidro de fundo chato $(8,5 \times 2,4 \mathrm{~cm})$, e alimentadas com uma gotícula de mel. Para cada fêmea foram oferecidos 15-20 ovos, de cada hospedeiro, com até 24 horas de idade. Os ovos foram substituídos diariamente, tendo-se procedido assim até a morte da fêmea. Os tubos foram mantidos em sala climatizada a $25 \pm 2^{\circ} \mathrm{C}, 70 \pm 10 \%$ de UR e 12 horas de fotófase. A elaboração da tabela de vida de fertilidade foi baseada em Silveira-Neto et al. (1976), na qual, com base nos dados de parasitismo diário durante a fase adulta, calculou-se o tempo médio de uma geração (T), a taxa líquida de reprodução (Ro) - a qual indica o número de vezes que a população poderá se multiplicar por geração -, a taxa intrínseca de crescimento populacional $\left(\mathrm{r}_{\mathrm{m}}\right)$ e a taxa finita de crescimento populacional ( $\lambda$ ). As médias foram comparadas pelo teste $t$ unilateral a $5 \%$ de significância de acordo com procedimento descrito por Maia et al. 
(2000), com auxílio do programa estatístico SAS (SAS Institute, Cary, NC, EUA).

\section{Resultados e Discussão}

As fêmeas de T. galloi avaliadas nos testes com chance de escolha entre os ovos de D. flavipennella e $D$. saccharalis apresentaram maior parasitismo em ovos do hospedeiro D. flavipennella. Com relação aos dados de viabilidade e longevidade, não houve diferença entre os hospedeiros testados (Tabela 1). Nos testes sem chance de escolha, nos quais os ovos foram oferecidos separadamente às fêmeas de T. galloi, não houve diferença nos parâmetros parasitismo, viabilidade e longevidade.

Amelhor aceitação do parasitoide T. galloi em relação a ovos de D. flavipennella foi evidenciada pela maior taxa de parasitismo nesse hospedeiro. As características físico-químicas do hospedeiro podem afetar a aceitação e a adaptação da espécie e/ou linhagem do parasitoide, o que pode interferir nas características biológicas e na sua agressividade (capacidade de parasitismo) e, assim, comprometer sua qualidade e eficiência (Pratissoli et al., 2010). A preferência das fêmeas de Trichogramma spp. está relacionada com a qualidade nutricional do hospedeiro escolhido para o parasitismo e daquele no qual foram multiplicadas (Thuler et al., 2007). Além disso, a diferença na percentagem de parasitismo pode estar atrelada às características intrínsecas de cada espécie, ou até mesmo à espessura e à dureza do córion do ovo (Pak et al., 1990).

$\mathrm{Na}$ literatura, não foram encontradas informações acerca da qualidade nutricional de ovos de espécies do gênero Diatraea spp.; além disso, nos testes realizados, o parasitoide foi criado no hospedeiro A. kuehniella, não sendo, também, o condicionamento pré-imaginal das fêmeas de $T$. galloi a causa da preferência hospedeira registrada neste estudo.

Com relação aos valores de viabilidade dos ovos parasitados nos testes com e sem chance de escolha, os resultados indicaram que ambas as espécies hospedeiras são adequadas ao parasitoide, uma vez que permitiram seu completo desenvolvimento (Tabela 1). Os valores de longevidade dos parasitoides apresentaram média próxima a 8 dias para ambos os hospedeiros nos dois testes estudados. Valores inferiores foram encontrados por Pereira-Barros et al. (2005), que registraram longevidade média de 6,36 dias quando este mesmo parasitoide foi criado em ovos de D. saccharalis. Valores acima de 7 dias são considerados satisfatórios (Van Lenteren, 1994).

A capacidade de parasitismo e a longevidade são importantes parâmetros biológicos para determinação do potencial do parasitoide como agente de controle biológico. Nos testes realizados, esses parâmetros apresentaram valores adequados ao desenvolvimento de T. galloi sobre as espécies hospedeiras testadas. O número de adultos emergidos por ovo dos hospedeiros, bem como a razão sexual e o período de desenvolvimento,

Tabela 1. Média \pm erro-padrão do parasitismo, da viabilidade e da longevidade de Trichogramma galloi em ovos de Diatraea flavipennella e Diatraea saccharalis em testes com e sem chance de escolha ${ }^{(1)}$.

\begin{tabular}{|c|c|c|c|c|c|c|}
\hline \multirow[t]{2}{*}{ Parâmetro } & \multicolumn{2}{|c|}{ Com escolha } & \multirow[t]{2}{*}{$\mathrm{F}$} & \multicolumn{2}{|c|}{ Sem escolha } & \multirow[t]{2}{*}{$\mathrm{F}$} \\
\hline & D. flavipennella & D. saccharalis & & D. flavipennella & D. saccharalis & \\
\hline Parasitismo (\%) & $84,38 \pm 4,30 \mathrm{a}$ & $67,79 \pm 5,14 b$ & 26,39 & $80,40 \pm 4,99 \mathrm{ab}$ & $81,88 \pm 3,88 \mathrm{a}$ & 33,14 \\
\hline Viabilidade (\%) & $83,64 \pm 1,94 a$ & $81,78 \pm 1,88 \mathrm{a}$ & 29,16 & $78,67 \pm 1,70 \mathrm{a}$ & $73,22 \pm 1,43 a$ & 30,19 \\
\hline Longevidade (dias) & $8,14 \pm 0,20 \mathrm{a}$ & $8,13 \pm 0,17 \mathrm{a}$ & 31,02 & $8,13 \pm 0,17 \mathrm{a}$ & $8,07 \pm 0,18 \mathrm{a}$ & 37,03 \\
\hline
\end{tabular}

${ }^{(1)}$ Médias seguidas de letras iguais nas linhas não diferem pelo teste $\mathrm{F}$, a $5 \%$ de probabilidade.

Tabela 2. Média \pm erro-padrão de número de adultos por ovo do hospedeiro, razão sexual e período de desenvolvimento de Trichogramma galloi em ovos de Diatraea flavipennella e D. saccharalis em testes com e sem chance de escolha ${ }^{(1)}$.

\begin{tabular}{|c|c|c|c|c|c|c|}
\hline \multirow[t]{2}{*}{ Parâmetro } & \multicolumn{2}{|c|}{ Com escolha } & \multirow[t]{2}{*}{$\mathrm{F}$} & \multicolumn{2}{|c|}{ Sem escolha } & \multirow[t]{2}{*}{$\mathrm{F}$} \\
\hline & D. flavipennella & D. saccharalis & & D. flavipennella & D. saccharalis & \\
\hline Número de adultos por ovo & $2,26 \pm 0,05 a$ & $2,20 \pm 0,06 \mathrm{a}$ & 37,62 & $2,17 \pm 0,03 \mathrm{a}$ & $2,21 \pm 0,04 a$ & 35,05 \\
\hline Razão sexual & $0,84 \pm 0,01 \mathrm{a}$ & $0,72 \pm 0,06 \mathrm{a}$ & 41,12 & $0,76 \pm 0,08 \mathrm{a}$ & $0,83 \pm 0,03 \mathrm{a}$ & 43,01 \\
\hline Período (dias) & $9,63 \pm 0,14 a$ & $10,00 \pm 0,00 \mathrm{a}$ & 19,04 & $9,85 \pm 0,19 \mathrm{a}$ & $9,60 \pm 0,08 \mathrm{a}$ & 16,07 \\
\hline
\end{tabular}

${ }^{(1)}$ Médias seguidas de letras iguais nas linhas não diferem entre si pelo teste $\mathrm{F}$, a $5 \%$ de probabilidade. 
também não apresentaram diferenças entre as duas espécies hospedeiras nos testes com e sem chance de escolha (Tabela 2). A razão sexual dos descendentes de $T$. galloi criados em ovos de D. flavipennella e D. saccharalis atingiu o índice aceitável exigido no controle de qualidade de Trichogramma (Prezotti \& Parra, 2002), sendo os valores superiores a 0,5 . O sexo da progênie pode ser influenciado pela qualidade do hospedeiro bem como pela ação da bactéria do gênero Wolbachia (Rodrigues, 2013).

A taxa de parasitismo decresceu em ambos os hospedeiros com o avanço na idade da fêmea de T. galloi. Registrou-se parasitismo superior a $80 \%$ em ovos parasitados por fêmea com até 24 horas de idade e, em ovos parasitados por fêmeas com até 72 horas, o parasitismo foi inferior a $30 \%$. Em relação à viabilidade dos ovos parasitados, não houve diferença entre as espécies hospedeiras e nem entre as idades das fêmeas (Tabela 3), tendo esses resultados também sido observados por Polanczyk et al. (2007) com T. exiguum sobre ovos de Plutella xylostella L. (Lepidoptera: Plutellidae).

Em espécies de Trichogramma, as fêmeas, quando jovens, apresentam maior taxa de oviposição, a qual se reduz gradativamente com a idade (Pratissoli et al., 2004; Zago et al., 2006). Tais observações também foram registradas por Pratissoli et al. (2007), que estudaram as diferentes idades de fêmeas de T. pretiosum sobre ovos de $P$. xylostella e observaram redução na capacidade de parasitismo dessa espécie. Zago et al. (2007) também registraram um decréscimo no parasitismo de Trichogramma pratissolii Querino \& Zucchi sobre A. kuehniella e Corcyra cephalonica

Tabela 3. Média \pm erro-padrão da taxa de parasitismo e da viabilidade de Trichogramma galloi em ovos de Diatraea flavipennella e Diatraea saccharalis no período de 24, 48 e 72 horas após emergência da fêmea.

\begin{tabular}{lcccc}
\hline Hospedeiro & \multicolumn{3}{c}{ Período em horas } & F \\
\cline { 2 - 3 } & \multicolumn{4}{c}{48} \\
\hline \multicolumn{5}{c}{ Taxa de parasitismo (\%) } \\
D. flavipennella & $89,44 \pm 4,29 \mathrm{Aa}$ & $49,96 \pm 3,65 \mathrm{Ab}$ & $24,55 \pm 3,31 \mathrm{Ac}$ & 9,06 \\
D. saccharalis & $84,91 \pm 3,68 \mathrm{Aa}$ & $43,32 \pm 3,22 \mathrm{Ab}$ & $28,46 \pm 3,96 \mathrm{Ac}$ & 8,40 \\
\hline \multicolumn{5}{c}{ Viabilidade (\%) } \\
D. flavipennella & $79,24 \pm 3,69 \mathrm{Aa}$ & $74,09 \pm 3,99 \mathrm{Aa}$ & $71,13 \pm 3,05 \mathrm{Aa}$ & 26,13 \\
D. saccharalis & $81,16 \pm 3,11 \mathrm{Aa}$ & $78,27 \pm 3,89 \mathrm{Aa}$ & $71,47 \pm 3,67 \mathrm{Aa}$ & 21,11 \\
\hline
\end{tabular}

${ }^{(1)}$ Médias seguidas de letras iguais, minúsculas nas linhas e maiúsculas nas colunas, não diferem pelo teste de Tukey e pelo teste F, respectivamente, a $5 \%$ de probabilidade.
(Stainton) (Lepidoptera: Pyralidae), em função da idade das fêmeas.

Fêmeas de inimigos naturais possuem a capacidade de reabsorver seus oócitos com o aumento da idade, o que pode acarretar redução no parasitismo e na vida fértil. Tal fenômeno é considerado comum em insetos e pode ser influenciado por hormônios ecdisteroides ou pela ausência de hospedeiros para oviposição (Bodin et al., 2009).

O decréscimo na taxa de parasitismo demonstra a relevância da idade do parasitoide, além do período de desenvolvimento embrionário do hospedeiro, na qualidade de $T$. galloi em uma criação massal, bem como nas liberações de campo para o controle biológico de pragas.

O período de desenvolvimento, número de adultos por ovo do hospedeiro, razão sexual e longevidade das fêmeas não apresentaram diferenças entre os hospedeiros (Tabela 4). O número de adultos por ovo variou de 2,18 a 2,20. Esses resultados indicam que em ovos de D. flavipennella e D. saccharalis, houve quantidade suficiente de nutrientes para suportar o desenvolvimento de mais de um parasitoide por ovo hospedeiro, sem interferir na qualidade do inseto produzido.

A razão sexual foi de cerca de $70 \%$ de fêmeas para ambas as espécies hospedeiras, o que evidenciou boa adequação dos ovos de Diatraea spp. ao parasitoide. A longevidade das fêmeas apresentou um período médio de 9 dias, tendo esse valor sido considerado satisfatório. $\mathrm{O}$ efeito da idade das fêmeas sobre a longevidade dos seus descendentes também foi registrado por Pratissoli et al. (2007), que estudaram o efeito das diferentes idades de fêmeas de $T$. pretiosum sobre ovos de $P$. xylostella, e observaram que estas afetam a capacidade de parasitismo e longevidade dos descendentes dessa

Tabela4. Média \pm erro-padrãodoperíododedesenvolvimento, número de adultos por ovo do hospedeiro, razão sexual e longevidade das fêmeas de Trichogramma galloi em ovos de Diatraea flavipennella e Diatraea saccharalis ${ }^{(1)}$.

\begin{tabular}{lccc}
\hline Parâmetro & D. flavipennella & D. saccharalis & $\mathrm{F}$ \\
\hline Período (dias) & $9,82 \pm 0,31 \mathrm{a}$ & $9,87 \pm 0,28 \mathrm{a}$ & 39,01 \\
$\mathrm{~N}^{\mathrm{o}}$ de adultos por ovo & $2,18 \pm 0,08 \mathrm{a}$ & $2,20 \pm 0,05 \mathrm{a}$ & 36,42 \\
Razão sexual & $0,78 \pm 0,03 \mathrm{a}$ & $0,79 \pm 0,05 \mathrm{a}$ & 38,20 \\
Longevidade & $8,41 \pm 0,14 \mathrm{a}$ & $8,52 \pm 0,16 \mathrm{a}$ & 42,13 \\
\hline \multicolumn{4}{l}{${ }^{(1)}$ Médias seguidas de letras iguais nas linhas não diferem pelo teste $\mathrm{F}$, a $5 \%$} \\
de probabilidade.
\end{tabular}

Pesq. agropec. bras., Brasília, v.51, n.4, p.293-300, abr. 2016 DOI: 10.1590/S0100-204X2016000400001 
espécie de parasitoide. Polanczyk et al. (2007) também registraram alteração na longevidade dos descendentes de $T$. exiguum, criados em ovos de P. xylostella, conforme a idade da fêmea.

Segundo Pratissoli et al. (2007), parasitoides mais longevos podem apresentar maior eficiência em campo, uma vez que possuem maior tempo para procurar e parasitar ovos dos seus hospedeiros, contribuindo, assim, para o aumento da sua população e da eficácia no controle de surtos posteriores de pragas. Por outro lado, a capacidade total de parasitismo deve ser conhecida, pois se esta for concentrada nos primeiros dias, não haverá necessidade de indivíduos mais longevos em campo (Dias et al., 2008).

A adequação dos ovos de D. flavipennella e $D$. saccharalis ao desenvolvimento de $T$. galloi foi confirmada pelo período de desenvolvimento, número de adultos por ovo, razão sexual e longevidade das fêmeas, os quais apresentaram valores satisfatórios para o desenvolvimento do parasitoide, uma vez que estão de acordo com os índices recomendados (Prezotti \& Parra, 2002). Os índices médios recomendados como padrão de qualidade pela Organização Internacional de Controle Biológico (IOBC) para parasitoides do gênero Trichogramma são: parasitismo $\geq 25$ ovos/ fêmea em 48 horas; emergência $\geq 80 \%$; razão sexual $\geq$ 0,5; e longevidade $\geq 7$ dias (Van Lenteren, 1994).

A sobrevivência das fêmeas provenientes de ambas as espécies não apresentou diferença (Figura 1).

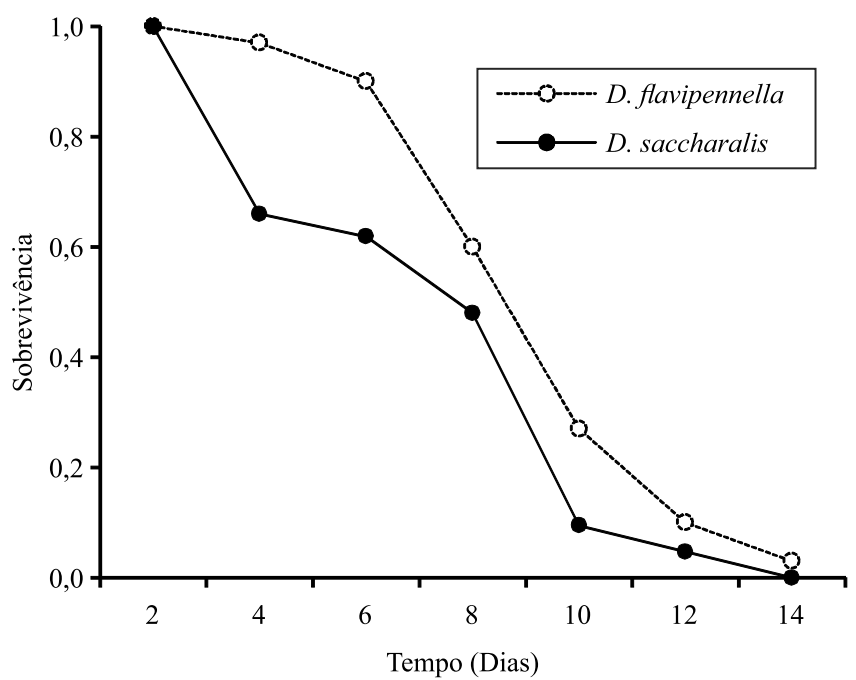

Figura 1. Curvas de sobrevivência de fêmeas de Trichogramma galloi obtidas de ovos de Diatraea flavipennella e Diatraea saccharalis.
A tabela de vida e fertilidade indicou duração média (T) de 12 dias e compreendeu o período entre o nascimento dos indivíduos de uma geração e o da geração seguinte (Tabela 5). Valores próximos a esse foram registrados por Dias et al. (2010) em ovos de A. kuehniella parasitados por Trichogrammatoidea annulata De Santis, 1972; e registrados por Meira et al. (2011) em ovos de P. xylostella parasitados por Trichogramma acacioi Brun, Moraes \& Soares; Trichogramma atopovirilia Oatman \& Platner; Trichogramma exiguum Pinto \& Platner (Hymenoptera: Trichogrammatidae); e T. pretiosum.

A taxa líquida reprodutiva $\left(\mathrm{R}_{0}\right)$ de $T$. galloi sobre ovos dos hospedeiros D. flavipennella e D. saccharalis variou de 23,71 a 28,34 . As taxas intrínsecas de crescimento populacional $\left(\mathrm{r}_{\mathrm{m}}\right)$ variaram de 0,24 a 0,26 entre $D$. saccharalis e $D$. flavipennella, respectivamente; e as taxas finitas $(\lambda)$, variaram de 1,29 a 1,31 entre $D$. saccharalis e $D$. flavipennella, respectivamente. Pratissoli et al. (2007) encontraram valores de $r_{m}$ e $\lambda$ próximos aos registrados neste estudo, para $25{ }^{\circ} \mathrm{C}$, ao elaborarem tabela de vida e fertilidade de T. pretiosum sobre ovos de Tuta absoluta (Meyrick) (Lepidoptera: Gelechiidae). A taxa finita de crescimento populacional $(\lambda)$ representa o número de fêmeas adicionadas à população e está relacionada diretamente com a taxa líquida reprodutiva $\left(\mathrm{R}_{0}\right)$ e com a taxa intrínseca de crescimento populacional $\left(\mathrm{r}_{\mathrm{m}}\right)$, sendo estes os principais parâmetros que influenciam no valor da $\lambda$.

O conhecimento acerca do comportamento de T. galloi sobre as duas espécies de Diatraea fornece informações sobre o seu potencial de controle. Sendo assim, o estudo atual mostrou que T. gallo $i$

Tabela 5. Duração média de cada geração (T), taxa líquida reprodutiva $\left(\mathrm{R}_{0}\right)$, taxa intrínseca de crescimento populacional $\left(\mathrm{r}_{\mathrm{m}}\right)$ e taxa finita de crescimento populacional ( $\lambda$ ) de Trichogramma galloi parasitando ovos de Diatraea flavipennella e Diatraea saccharalis ${ }^{(1)}$.

\begin{tabular}{lcccc}
\hline Hospedeiro & $\begin{array}{c}\mathrm{T} \\
\text { (dias) }\end{array}$ & $\begin{array}{c}\mathrm{R}_{0} \\
\text { (fêmeas } \\
\text { por fêmeas) }\end{array}$ & $\begin{array}{c}\mathrm{r}_{\mathrm{m}} \\
\text { fềmea por dia) }\end{array}$ & $\begin{array}{c}\lambda \\
\text { (fêmea por } \\
\text { dia) }\end{array}$ \\
\hline \multirow{2}{*}{ D. flavipennella } & $12,21 \mathrm{a}$ & $23,71 \mathrm{a}$ & $0,26 \mathrm{a}$ & $1,31 \mathrm{a}$ \\
& $(9,57-13,31)^{(2)}$ & $(19,68-29,17)$ & $(0,21-0,33)$ & $(1,24-1,34)$ \\
D. saccharalis & $12,39 \mathrm{a}$ & $28,34 \mathrm{a}$ & $0,24 \mathrm{a}$ & $1,29 \mathrm{a}$ \\
& $(9,43-13,23)$ & $(23,14-32,04)$ & $(0,17-0,28)$ & $(1,23-1,31)$ \\
\hline
\end{tabular}

${ }^{(1)}$ Médias seguidas de letras igauis nas colunas não diferem pelo teste t a $5 \%$ de probabilidade. ${ }^{(2)}$ Intervalo de confiança a $95 \%$, entre parênteses. 
também apresentou um eficiente desempenho sobre a espécie D. flavipennella. Verificou-se, também, que tanto $D$. flavipennella quanto $D$. saccharalis são eficientemente parasitados por T. galloi, sendo este um agente com grande potencial para ser utilizado em programas de manejo integrado dessas espécies. Contudo, ainda há necessidade de dar prosseguimento a este estudo, para comprovação do desempenho do parasitoide T. galloi em condições de semicampo e campo. Sabe-se que o desempenho do parasitoide em campo depende das características fenológicas, bem como da arquitetura da planta. A dinâmica da praga, condições climáticas e estratégias de liberação também são fatores a serem considerados em pesquisas futuras (Gingras et al., 2002).

\section{Conclusões}

1. O parasitoide Trichogramma galloi apresenta melhor aceitação de ovos de Diatraea flavipennella em testes com chance de escolha.

2. As brocas $D$. flavipennella e D. saccharalis são hospedeiros igualmente adequados ao parasitismo por T. galloi.

3. Os valores da tabela de vida de fertilidade indicam capacidade do parasitoide $T$. galloi em adicionar indivíduos por fêmea por dia nos hospedeiros D. flavipennella e D. saccharalis.

\section{Agradecimentos}

À Fundação de Amparo à Pesquisa do Estado de Alagoas (Fapeal, processo PEB-20110325-002-00040041), pela concessão de bolsa.

\section{Referências}

BODIN, A.; JALOUX, B.; DELBECQUE, J.-P.; VANNIER, F.; MONGE, J.-P.; MONDY, N. Reproduction in a variable environment: how does Eupelmus vuilleti, a parasitoid wasp, adjust oogenesis to host availability? Journal of Insect Physiology, v.55, p.643-648, 2009. DOI: 10.1016/j.jinsphys.2009.04.003.

BOTELHO, P.S.M.; PARRA, J.R.P.; CHAGAS NETO, J.F.; OLIVEIRA, C.P.B. Associação do parasitóide de ovos Trichogramma galloi Zucchi (Hymenoptera: Trichogrammatidae) e do parasitóide larval Cotesia flavipes (Cam.) (Hymenoptera: Braconidae) no controle de Diatraea saccharalis, (Fabr.) (Lepidoptera: Crambidae) em cana-de-açúcar. Anais da Sociedade Entomológica do Brasil, v.28, p.491-496, 1999. DOI: 10.1590/ s0301-80591999000300015.
CHEAVEGATTI-GIANOTTO, A.; ABREU, H.M.C. de; ARRUDA, P.; BESPALHOK FILHO, J.C.; BURNQUIST, W.L.; CRESTE, S.; DI CIERO, L.; FERRO, J.A. FIGUEIRA, A.V. de O.; FILGUEIRAS, T. de S.; GROSSI-DE-SÁ, M. de F.; GUZZO, E.C.; HOFFMANN, H.P.; LANDELL, M.G. de A.; MACEDO, N.; MATSUOKA, S.; REINACH, F. de C.; ROMANO, E.; SILVA, W.J. da; SILVA FILHO, M. de C.; ULIAN, E.C. Sugarcane (Saccharum $x$ officinarum): A reference study for the regulation of genetically modified cultivars in Brazil. Tropical Plant Biology, v.4, p.62-89, 2011. DOI: 10.1007/ s12042-011-9068-3.

DIAS, N. da S.; PARRA, J.R.P.; DIAS, C.T. dos S. Tabela de vida de fertilidade de três espécies neotropicais de Trichogrammatidae em ovos de hospedeiros alternativos como critério de seleção hospedeira. Revista Brasileira de Entomologia, v.54, p.120-124, 2010. DOI: 10.1590/S0085-56262010000100016.

DIAS, N. da S.; PARRA, J.R.P.; LIMA, T.C. da C. Seleção de hospedeiro alternativo para três espécies de tricogramatídeos neotropicais. Pesquisa Agropecuária Brasileira, v.43, p.1467-1473, 2008. DOI: 10.1590/S0100-204X2008001100004.

DINARDO-MIRANDA, L.L. In: DINARDO-MIRANDA, L.L.; VASCONCELOS, A.C.M. de; LANDELL, M.G. de A. (Ed.). Cana-de-açúcar. Campinas: Instituto Agronômico, 2008. p.349-404.

DINARDO-MIRANDA, L.L.; ANJOS, I.A. dos; COSTA, V.P. da; FRACASSO, J.V. Resistance of sugarcane cultivars to Diatraea saccharalis. Pesquisa Agropecuária Brasileira, v.47, p.1-7, 2012. DOI: 10.1590/S0100-204X2012000100001.

DINARDO-MIRANDA, L.L.; VASCONCELOS, A.C.M. de; LANDELL, M.G. de A. Cana-de-açúcar. Campinas: Instituto Agronômico, 2010. 882p.

FREITAS, M. do R.T. de; SILVA, E.L. da; MENDONÇA, A. de L.; SILVA, C.E. da; FONSECA, A.P.P. da; MENDONÇA, A. de L.; SANTOS, J. de S.; NASCIMENTO, R.R. do; SANT'ANA, A.E.G. The biology of Diatraea flavipennella (Lepidoptera: Crambidae) reared under laboratory conditions. Florida Entomologist, v.90, p.309-313, 2007. DOI: 10.1653/0015-4040(2007)90[309:TBODF L]2.0.CO;2.

GINGRAS, D.; DUTILLEUL, P.; BOIVIN, G. Modeling the impact of plant structure on host-finding behavior of parasitoids. Oecologia, v.130, p.396-402, 2002. DOI: 10.1007/s00442-001-0819-y.

HASSAN, S.A. Seleção de espécies de Trichogramma para o uso em programas de controle biológico. In: PARRA, J.R.P.; ZUCCHI, R.A. (Ed.). Trichogramma e o controle biológico aplicado. Piracicaba: Fealq, 1997. p.183-205.

LIMA, H.M.A.; RODRIGUES, V. de M.; DUARTE, A.G.; ARAUJO JÚNIOR, J.V. de; LOPES, D.O.P.; LIMA, I.S. de; DUARTE, A.G.; TEODORO, I. Infestação de Diatraea spp. (LEPIDOPTERA: CRAMBIDAE) em variedades RB (República do Brasil) de cana-de-açúcar. Comunicata Scientiae, v.4, p.407-413, 2013.

MACEDO, N. Método de criação do parasitóide Cotesia flavipes (Cameron, 1891). In: BUENO, V.H.P. (Ed.). Controle biológico de pragas: produção massal e controle de qualidade. Lavras: UFLA, 2002. p.161-175. 
MAIA, A. de H.N.; LUIZ, A.J.B.; CAMPANHOLA, C. Statistical Inference on Associated Fertility Life Table Parameters Using Jackknife Technique: Computational Aspects. Journal of Economic Entomology, v.93, p.511-518, 2000. DOI: 10.1603/0022-0493-93.2.511.

MEIRA, A.L.; PRATISSOLI, D.; SOUZA, L.P. de; STURM, G. Seleção de espécies de Trichogramma sp. em ovos da traça-das-crucíferas, Plutella xylostella. Revista Caatinga, v.24, p.1-8, 2011.

OLIVEIRA, H.N. de; SANTANA, D.R.S.; BELLON, P.P.; OLIVEIRA, F.C. de. Influência da idade dos ovos de Diatraea saccharalis (Lepidoptera: Crambidae) no parasitismo de Trichogramma galloi (Hymenoptera: Trichogrammatidae). Interciência, v.39, p.46-48, 2014.

PAK, G.A.; DALEN, A. van; KAASHOEK, N.; DIJKMAN, H. Host egg chorion structure influencing host suitability for the egg parasitoid Trichogramma Westwood. Journal of Insect Physiology, v.36, p.247-258, 1990. DOI: 10.1016/0022-1910(90)90174-E.

PEREIRA-BARROS，J.L.; BROGLIO-MICHELETTI， S.M.F.; SANTOS,A.J.N.dos;CARVALHO,L.W.T.de;CARVALHO,L.H.T. de; OLIVEIRA, C.J.T.de. Aspectos biológicos de Trichogramma galloi Zucchi, 1988 (Hymenoptera: Trichogrammatidae) criados em ovos de Diatraea saccharalis (Fabricius, 1794) (Lepidoptera: Crambidae). Ciência e Agrotecnologia, v.29, p.714-718, 2005. DOI: $10.1590 /$ S1413-70542005000400002.

POLANCZYK, R.A.; PRATISSOLI, D.; HOLTZ,A.M.; PEREIRA, C.L.T.; FURTADO, I.S. de A. Efeito da idade de Trichogramma exiguum e do desenvolvimento embrionário da traça-das-crucíferas sobre as características biológicas do parasitóide. Acta Scientiarum. Biological Sciences, v.29, p.161-166, 2007. DOI: 10.4025/actascibiolsci.v29i2.522.

PRATISSOLI, D.; DALVI, L.P.; POLANCZYK, R.A.; ANDRADE, G.S.; HOLTZ, A.M.; NICOLINE, H.O. Características biológicas de Trichogramma exiguum em ovos de Anagasta kuehniella e Sitotroga cerealella. Idesia (Chile), v.28, p.39-42, 2010. DOI: 10.4067/s0718-34292010000100006.

PRATISSOLI, D.; POLANCZYK, R.A.; ANDRADE， G.S.; HOLTZ, A.M.; SILVA, A.F. da; PASTORI, P.L. Tabela de vida de fertilidade de cinco linhagens de Trichogramma pretiosum Riley (Hym.: Trichogrammatidae) criadas em ovos de Tuta absoluta (Merick) (Lep.: Gelechiidae), sob temperaturas constantes e alternadas. Ciência Rural, v.37, p.618-622, 2007. DOI: 10.1590/ S0103-84782007000300003.
PRATISSOLI, D.; ZANUNCIO, J.C.; VIANNA, U.R.; ANDRADE, J. de S.; GUIMARÃES, E.M.; ESPINDULA, M.C. Fertility life Table of Trichogramma pretiosum and Trichogramma acacioi on eggs of Anagasta kuehniella at different temperatures. Pesquisa Agropecuária Brasileira, v.39, p.193-196, 2004. DOI: 10.1590/S0100-204X2004000200014.

PREZOTTI, L.; PARRA, J.R.P. Controle de qualidade em criações massais de parasitóides e predadores. In: PARRA, J.R.P.; BOTELHO, P.S.M.; CORREAA-FERREIRA, B.S.; BENTO, J.M.S. (Ed.). Controle biológico no Brasil: parasitóides e predadores. São Paulo: Manole, 2002. p.295-311.

RODRIGUES, M.S. Wolbachia, una pandemia con posibilidades. Revista de la Sociedad Entomológica Argentina, v.72, p.117-137, 2013.

SILVEIRA-NETO, S.; NAKANO, O.; BARBIN, D.; VILLANOVA, N.A. Manual de ecologia dos insetos. São Paulo: Agronômica Ceres, 1976. 519p.

SIQUEIRA, J.R.; BUENO, R.C.O. de F.; BUENO, A. de F.; VIEIRA, S.S. Preferência hospedeira do parasitoide de ovos Trichogramma pretiosum, Ciência Rural, v.42, p.1-5, 2012. DOI: 10.1590/S0103-84782011005000156.

THULER, R.T.; VOLPE, H.X.L.; DE BORTOLI, S.A.; GOULART, R.M.; VIANA, C.L. T.P. Metodologia para avaliação da preferência hospedeira de parasitóides do gênero Trichogramma Westood. Boletín de Sanidad Vegetal. Plagas, v.33, p.333-340, 2007.

VAN LENTEREN, J.C. Designing and implementing quality control of beneficial insects: towards more reliable biological pest control. Sting. Newsletter on Biological Control in Greenhouses, v.14, p.3-24, 1994.

VASCONCELOS, Y. Inseto contra inseto. Revista Pesquisa FAPESP, v.195, p.68-73, 2012.

ZAGO, H.B.; PRATISSOLI, D.; BARROS, R.; GONDIM JR., M.G.C. Biologia e exigências térmicas de Trichogramma pratissolii Querino \& Zucchi (Hymenoptera: Trichogrammatidae) em hospedeiros alternativos. Neotropical Entomology, v.35, p.377-381, 2006. DOI: 10.1590/S1519-566X2006000300013.

ZAGO, H.B.; PRATISSOLI, D.; BARROS, R.; GONDIM JR., M.G.C.; SANTOS JR., H.J.G. dos. Capacidade de parasitismo de Trichogramma pratissolii Querino \& Zucchi (Hymenoptera: Trichogrammatidae) em hospedeiros alternativos, sob diferentes temperaturas. Neotropical Entomology, v.36, p.84-89, 2007. DOI: 10.1590/S1519-566X2007000100010.

Recebido em 13 de maio de 2015 e aprovado em 10 de fevereiro de 2016 